\title{
Nonlinear fiber-optic strain sensor based on four-wave mixing in microstructured optical fiber
}

Gu, Bobo; Yuan, Scott Wu; Frosz, Michael H.; Zhang, A. Ping; He, Sailing; Bang, Ole

Published in:

Optics Letters

Link to article, DOI:

10.1364/OL.37.000794

Publication date:

2012

Document Version

Publisher's PDF, also known as Version of record

Link back to DTU Orbit

Citation (APA):

Gu, B., Yuan, S. W., Frosz, M. H., Zhang, A. P., He, S., \& Bang, O. (2012). Nonlinear fiber-optic strain sensor based on four-wave mixing in microstructured optical fiber. Optics Letters, 37(5), 794-796.

https://doi.org/10.1364/OL.37.000794

\section{General rights}

Copyright and moral rights for the publications made accessible in the public portal are retained by the authors and/or other copyright owners and it is a condition of accessing publications that users recognise and abide by the legal requirements associated with these rights.

- Users may download and print one copy of any publication from the public portal for the purpose of private study or research.

- You may not further distribute the material or use it for any profit-making activity or commercial gain

- You may freely distribute the URL identifying the publication in the public portal 


\title{
Nonlinear fiber-optic strain sensor based on four-wave mixing in microstructured optical fiber
}

\author{
Bobo Gu, ${ }^{1,2, *}$ Wu Yuan, ${ }^{1}$ Michael H. Frosz, ${ }^{3}$ A. Ping Zhang, ${ }^{2}$ Sailing He, ${ }^{2}$ and Ole Bang ${ }^{1}$ \\ ${ }^{1}$ DTU Fotonik, Department of Photonics Engineering, Technical University of Denmark, \\ Ørsteds Plads 343, DK-2800 Kgs. Lyngby, Denmark \\ ${ }^{2}$ Center for Optical and Electromagnetic Research, State Key Laboratory of Modern Optical Instrumentation, \\ Zhejiang University, Hangzhou 310058, China \\ ${ }^{3}$ Max Planck Institute for the Science of Light, Günther-Scharowsky-Str.1, Building 24, 91058 Erlangen, Germany \\ *Corresponding author: gubobo@coer.zju.edu.cn
}

Received September 22, 2011; revised November 28, 2011; accepted December 6, 2011; posted December 7, 2011 (Doc. ID 155127); published February 21, 2012

\begin{abstract}
We demonstrate a nonlinear fiber-optic strain sensor, which uses the shifts of four-wave mixing Stokes and anti-Stokes peaks caused by the strain-induced changes in the structure and refractive index of a microstructured optical fiber. The sensor thus uses the inherent nonlinearity of the fiber and does not require any advanced postprocessing of the fiber. Strain sensitivity of $-0.23 \mathrm{pm} / \mu \varepsilon$ is achieved experimentally and numerical simulations reveal that for the present fiber the sensitivity can be increased to $-4.46 \mathrm{pm} / \mu \varepsilon$ by optimizing the pump wavelength and power. (C) 2012 Optical Society of America

OCIS codes: $\quad 060.2370,060.4005,060.4370,060.5295,190.4370,190.4380$.
\end{abstract}

Strain is an important physical parameter that needs to be monitored in many areas of engineering. Various fiber-optic schemes, including fiber gratings [1], MachZehnder interferometers [2]], Sagnac interferometers [3] and Fabry-Perot interferometers []], have been proposed and demonstrated for strain sensing. These sensors all use the linear properties of the fiber and rely on either specialty fibers, such as highly birefringent fibers, or postprocessing of the fibers, such as grating writing.

It has been proposed to instead use the Stokes and anti-Stokes lines generated by nonlinear degenerate four-wave mixing (FWM) [5] in a microstructured optical fiber (MOF) to achieve highly efficient sensing of materials infiltrated into the holes [6]. The fiber-optic FWM sensor technology can offer high sensitivity due to the extreme sensitivity of FWM to the dispersion profile [7] and it does not require fiber postprocessing. It was recently verified that highly sensitive refractive index sensing, and thus also biosensing, can indeed be achieved with the FWM technique $[6,8]$.

In this Letter we demonstrate experimentally a novel fiber-optic strain sensor based on FWM in a MOF. The operation principle is to track the Stokes wavelength $\lambda_{S}$ and/or anti-Stokes wavelength $\lambda_{\mathrm{aS}}$ (frequencies $\omega_{S}$ and $\omega_{\mathrm{aS}}$ ) generated by FWM under different strain, which changes the dispersion profile of the MOF as seen in Fig. 1. Compared with conventional fibers, the MOF allows tuning the zero-dispersion wavelength (ZDW) to be close to the pump wavelength for enhancing the FWM sensitivity. Here we choose a pump at $\lambda_{p}=$ $1064 \mathrm{~nm}$. We therefore use a MOF (NL-4.7-1030, NKT Photonics $A / S$ ) with a pitch of $\Lambda_{0}=3.15 \mu \mathrm{m}$, and a relative hole diameter of $d_{0} / \Lambda_{0}=0.5$, as shown in the inset of Fig. 1. This MOF has a mode-field diameter of $3.8 \mu \mathrm{m}$, a nonlinear coefficient of $\gamma=12 \mathrm{~W} \mathrm{~km}^{-2}$, and a loss of $<15 \mathrm{~dB} / \mathrm{km}$ at $1060 \mathrm{~nm}$. The dispersion profile, calculated with commercial simulation software COMSOL, is shown in Fig. 1 . The unstrained $\mathrm{ZDW}$ is $\lambda_{\mathrm{ZDW}}=$ $1027.5 \mathrm{~nm}$, which means that we pump in the anomalous dispersion regime.
The experimental setup is shown in Fig. 2. The pump is a diode pumped, passively Q-switched Nd:YAG microchip laser (SNP-13E, Teem Photonics) with specifications given in the figure. A half-wave plate controls the reflectivity of the polarization dependent mirrors $(M)$ and a polarizer $(P)$ is used as a variable attenuator. A second half-wave plate controls the polarization before the beam is coupled into the fiber with a microscope objective (MO). A beam splitter (BS) and a camera are used as an aid in coupling the beam into the fiber core while the position of the fiber is adjusted with an X-Y-Z stage (FS). Two other stages $(S)$ are used to strain the fiber.

The FWM gain is given by $g(\omega)=\left[\left(\gamma P_{0}\right)^{2}-(\kappa / 2)^{2}\right]^{1 / 2}$, where $\quad \kappa(\omega)=2 \beta\left(\omega_{p}\right)-\beta\left(\omega_{S}\right)-\beta\left(\omega_{a S}\right)-2 \gamma P_{0}$ is the phase-mismatch, $\gamma=n_{2} \omega_{p} /\left(c A_{\text {eff }}\right)$ is the nonlinear parameter, $n_{2}=2.6 \times 10^{-20} \mathrm{~m}^{2} / \mathrm{W}$ is the nonlinear-index of silica, $c$ is the speed of light in vacuum, $A_{\text {eff }}$ is the effective area, $P_{0}$ is the peak power of the pump laser, and $\beta(\omega)$ is the propagation constant at frequency $\omega$ [7]. The maximum FWM gain occurs at phase-matching, where

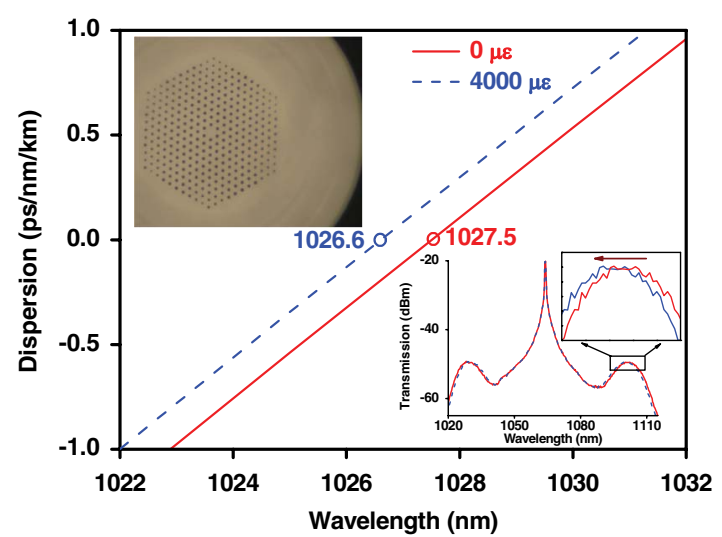

Fig. 1. (Color online) Calculated dispersion of the unstrained $(0 \mu \varepsilon$, solid) and strained (4000 $\mu \varepsilon$, dashed) MOF. Insets: fiber cross-section and experimental FWM spectra for $P_{0}=575 \mathrm{~W}$ with strain increasing from 0 to $4000 \mu \varepsilon$ along the arrow. 


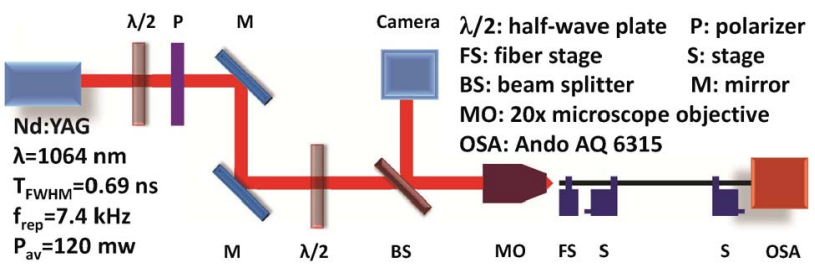

Fig. 2. (Color online) Sketch of the experimental setup.

$\kappa=0$. In the frequency domain this happens symmetrically around the pump at one or more sets of Stokes and anti-Stokes frequencies [9]. For our MOF there is only one set, whose wavelengths are calculated and shown as the solid curves of Fig. 3 .

The MOF is $1.2 \mathrm{~m}$ long and the peak power out of the laser is $P_{0}=P_{\mathrm{av}} /\left(T_{\mathrm{FWHM}} \cdot f_{\text {rep }}\right)=23.5 \mathrm{~kW}$, where $P_{\mathrm{av}}=$ $0.12 \mathrm{~W}$ is the average power, $T_{\mathrm{FWHM}}=0.69 \mathrm{~ns}$ is the pulse width, and $f_{\text {rep }}=7.4 \mathrm{kHz}$ is the repetition rate. To estimate the coupling efficiency from the MO to the MOF, we measured the power emitted from the MO and the MOF, respectively. Neglecting transmission loss of the only $1.2 \mathrm{~m}$ long MOF it was observed that the coupling efficiency is about $65 \%$.

We first investigate the FWM for different pump powers (measured out of the MOF) by rotating the polarizer and making sure that the power is low enough to avoid supercontinuum generation, in which case there would be no detectable FWM peaks. As presented in Fig. $\underline{3}$, the experimental and theoretical results agree well, both showing the expected increase (decrease) of the Stokes (anti-Stokes) wavelength with power [5]. The power dependence of the FWM peaks reveals that the MOF should be fixed tightly during the strain experiments to avoid changes in the coupling and subsequent measurement errors. We also investigated the influence of input polarization by rotating the second half-wave plate and found that the anti-Stokes and Stokes wavelengths did not change significantly. This means that the MOF has no significant birefringence and that the FWM strain sensor is independent of polarization.

When the MOF is imposed a longitudinal elongation $\Delta L$, one can write the axial strain as $\varepsilon=\Delta L / L$. The elasto-optic effect will induce a change in the refractive index

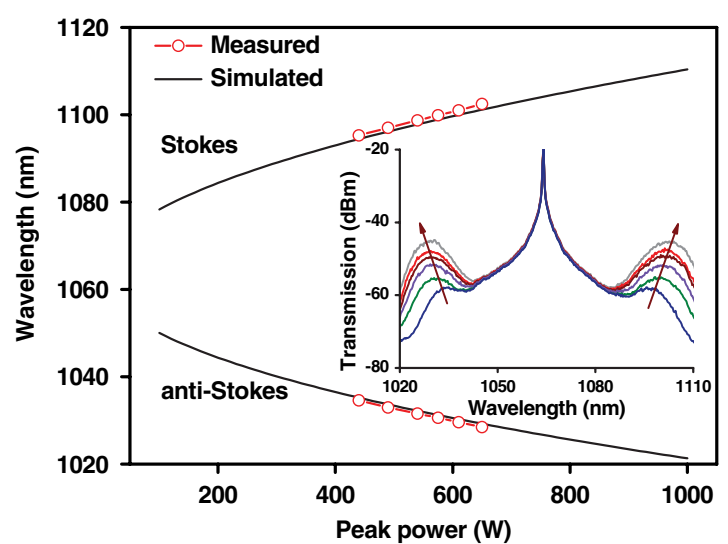

Fig. 3. (Color online) FWM peaks versus pump peak power. Inset: measured spectra with power increasing along the arrow. of $\Delta n=-n_{0}^{3}\left[p_{12}-\sigma\left(p_{11}+p_{12}\right)\right] \varepsilon / 2$, where $p_{11}$ and $p_{12}$ are strain-optic coefficients, and $\sigma$ is Poisson's ratio [10-12].

Although the unstrained refractive index of silica $n_{0}=$ $n_{0}(\omega)$ is well-known [5], the parameters $p_{11}, p_{12}$, and $\sigma$ are less known and depend on the fiber material, the drawing conditions, and the wavelength. In Table 1 we present three sets of parameters. Sets 1-2 are for a step-index silica fiber and set 3 is for bulk fused silica. When being elongated the cross section of the MOF will be compressed, which gives a geometrical change of the dispersion. We calculate it by using volume conservation and assuming that the strained fiber diameter $D$ is constant along the length, so that $D=D_{0} /(1+\varepsilon)^{1 / 2}$. We further assume that the change in hole diameter $d$ follows the change in fiber diameter, so that $d=d_{0} /(1+\varepsilon)^{1 / 2}$, and finally we assume that inside the MOF the hole diameter and pitch $\Lambda$ decrease in such a way that the relative hole diameter $d / \Lambda=d_{0} / \Lambda_{0}$ stays constant. $D_{0}, d_{0}$, and $\Lambda_{0}$ are the unstrained dimensions of the MOF.

The strain-induced structural and refractive index changes of the MOF will influence the dispersion profile and shift the ZDW from 1027.5 to $1026.6 \mathrm{~nm}$ for a strain of $4000 \mu \varepsilon$, as seen in Fig. 1. This will change the spectral location of the FWM peaks as seen in Fig. 1, which is the sensing principle of our nonlinear strain sensor. In our experimental study the polarizer and half-wave plates are fixed, so that the estimated input peak power is fixed at $575 \mathrm{~W}$. An $L=0.85 \mathrm{~m}$ long section of the MOF is clamped tightly between two translation stages, as shown in Fig. 2. One stage is fixed, while the other can move longitudinally to apply axial strain to the fiber manually with a displacement accuracy of $10 \mu \mathrm{m}$.

Figure 4(a) shows the measured and simulated spectral response of the FWM peaks versus the applied strain. The experiments show that when the strain is increased, the anti-Stokes peak shifts to longer wavelengths with a sensitivity of $0.20 \mathrm{pm} / \mu \varepsilon$, and the Stokes peak shifts to shorter wavelengths with a sensitivity of $-0.23 \mathrm{pm} / \mu \varepsilon$. Our simulations of Sets 1-3, taking both the geometrical and elasto-optic effect into account with a pump peak power of $575 \mathrm{~W}$, confirm the tendency, but with a lower sensitivity of about $0.08 \mathrm{pm} / \mu \varepsilon$ and $-0.09 \mathrm{pm} / \mu \varepsilon$ for the anti-Stokes and Stokes peaks, respectively.

In Fig. 4(b) we zoom on the numerical results for the Stokes peak to show how close the results for Sets 1-3 are. We also show two calculations with Set 1 (termed Set $1 \mathrm{a}$ and $1 \mathrm{~b}$ ). In Set 1a, only the elasto-optic effect is taken into account. Since the sensitivity for Set 1 is approximately twice that of Set 1a, the elasto-optically induced FWM shift is approximately the same as the geometrically induced FWM shift. This shows that the assumed geometry is important. In Set $1 \mathrm{~b}$ we therefore assume that the strained MOF has the more realistic

Table 1. Strain-Optic Coefficients $p_{11}$ and $p_{12}$, Poisson's Ratio $\sigma$, and Measurement Wavelength $\lambda$

\begin{tabular}{ccccrl}
\hline Parameters & $p_{11}$ & $p_{12}$ & \multicolumn{1}{c}{$\sigma$} & \multicolumn{1}{c}{$\lambda$} & Material \\
\hline Set $1[\underline{10}]$ & 0.113 & 0.252 & 0.16 & $633 \mathrm{~nm}$ & Fiber \\
Set 2 $[\overline{11}]$ & 0.120 & 0.27 & 0.25 & $1300 \mathrm{~nm}$ & Fiber \\
Set $3[\underline{\underline{12}}]$ & 0.124 & 0.28 & 0.164 & $644 \mathrm{~nm}$ & Bulk \\
\hline
\end{tabular}



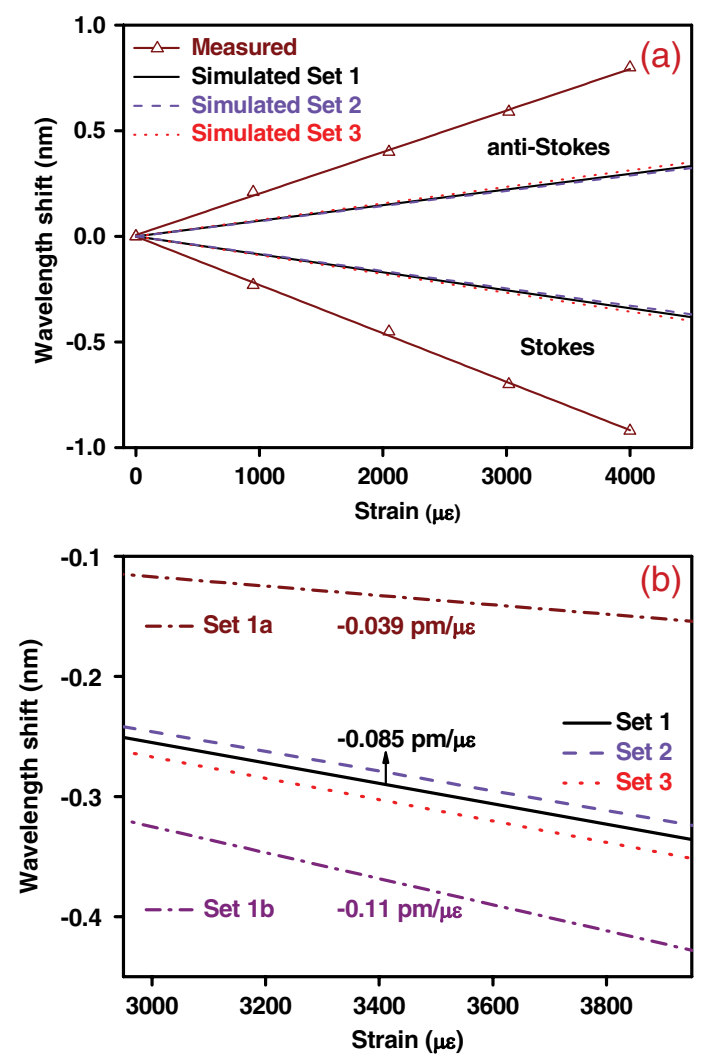

Fig. 4. (Color online) (a) Measured and simulated FWM peaks versus strain. (b) Zoom on Stokes peak for the simulations. Parameter Sets 1-3 are given in Table 1 . Sets 1a-b are explained in the text.

geometrical shape of a tapered fiber, i.e., a standard hyperboloid of one sheet. Using again volume conservation, the fiber diameter at the center of the strained section is then $D=D_{0}[(1-\varepsilon / 2) /(1+\varepsilon)]^{1 / 2}$, which is a factor of $[1-\varepsilon / 2]^{1 / 2}$ smaller than when using the constant diameter assumption. Using this reduced fiber diameter gives a sensitivity for Set $1 b$, which is increased to $-0.11 \mathrm{pm} / \mu \varepsilon$, as shown in Fig. 4(b). We believe that the other assumptions made in calculating the geometrical effect and the fact that the elasto-optic parameters are not for the pure silica MOF we use, and for wavelengths that differ significantly from $1064 \mathrm{~nm}$, can explain the difference of about a factor of 2 between the numerically and experimentally found sensitivities.

Using the present MOF as the sensing element, one can tune the wavelength and peak power of the pump laser to optimize the sensitivity []ㅡ. Figure $\underline{5}$ shows numerical calculations of the strain sensitivities based on parameter Set 1 and the taper geometry (Set $1 \mathrm{~b}$ ). The unstrained $\mathrm{ZDW}$ is marked with a vertical line. The results show that when the pump is in the normal dispersion region (close to the ZDW), the FWM strain sensor has the highest sensitivity. We also see that around the optimum pump wavelength the sensitivity increases when the pump peak power is decreased. However, because the FWM gain is proportional to the power [5], it cannot be decreased too much without loosing the signal. In our experiment, the minimum pump peak power, which could generate sufficiently visible FWM peaks over $1.2 \mathrm{~m}$, is about $450 \mathrm{~W}$. The numerical results predict that the maximum strain

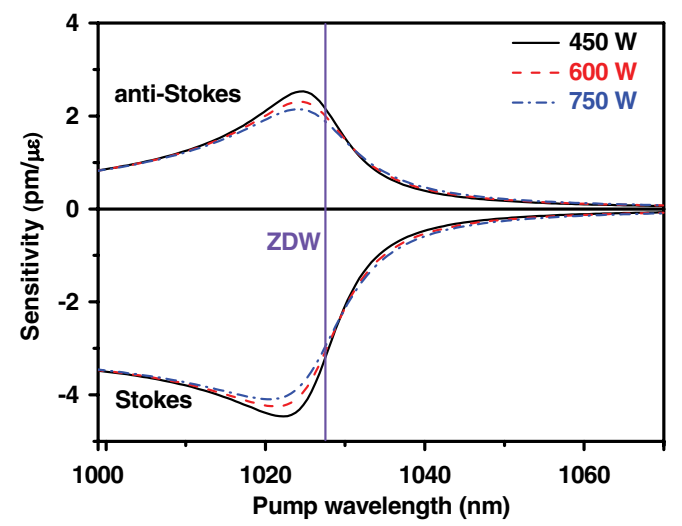

Fig. 5. (Color online) Sensitivity versus pump wavelength for different pump peak powers. Vertical line marks $\lambda_{\text {ZDW }}=1027.5 \mathrm{~nm}$.

sensitivity is $-4.46 \mathrm{pm} / \mu \varepsilon$ when it is pumped at $1022.3 \mathrm{~nm}$ with a peak power of $450 \mathrm{~W}$.

In conclusion, this is the first experimental work to demonstrate the feasibility of using FWM as the sensing principle for a fiber-optic strain sensor. This sensor is promising, because it relies on the inherent (nonlinear) properties of the fiber and does not require postprocessing. The experimentally demonstrated strain sensitivity is $-0.23 \mathrm{pm} / \mu \varepsilon$ for the Stokes wavelength, which equals that recently obtained for a MOF-based Sagnac strain sensor [3]. Numerical calculations predict that a sensitivity of $-\overline{4} .46 \mathrm{pm} / \mu \varepsilon$ can be achieved by optimizing the pump wavelength and power. This is more than four times higher than the sensitivity of FBG sensors at $1064 \mathrm{~nm}$ [1]. We expect that a further increase in sensitivity can be achieved by optimizing the MOF design. The FWM strain sensor will have a cross-sensitivity to temperature due to the thermo-optic coefficient of silica $\left(C_{T}=9 \times 10^{-6} / \mathrm{K}[\underline{1}]\right)$, just as the FBG sensor, which will be the subject of future investigations.

Bobo Gu would like to thank the support of the Chinese Scholar Council (CSC). The authors wish to thank Christian Agger and Johan R. Ott (DTU Fotonik) for helpful discussions on the dispersion calculations.

\section{References}

1. L. Rindorf and O. Bang, J. Opt. Soc. Am. B 25, 310 (2008).

2. Z. Tian and S. S.-H Yam, IEEE Photon. Technol. Lett. 21, 161 (2009).

3. X. Dong, H. Y. Tam, and P. Shum, Appl. Phys. Lett. 90, 151113 (2007).

4. S. Pevec and D. Donlagic, Opt. Express 19, 15641 (2011).

5. G. P. Agrawal, Nonlinear Fiber Optics, 4th ed. (Academic, 2007).

6. J. R. Ott, M. Heuck, C. Agger, P. D. Rasmussen, and O. Bang, Opt. Express 16, 20834 (2008).

7. M. H. Frosz, T. Sørensen, and O. Bang, J. Opt. Soc. Am. B 23, 1692 (2006).

8. M. H. Frosz, A. Stefani, and O. Bang, Opt. Express 19, 10471 (2011).

9. N. I. Nikolov, T. Sørensen, O. Bang, and A. Bjarklev, J. Opt. Soc. Am. B 20, 2329 (2003).

10. A. Bertholds and R. Dandliker, J. Lightwave Technol. 6, 17 (1988).

11. L. Yuan, Appl. Opt. 36, 6246 (1997).

12. N. F. Borelli and R. A. Miller, Appl. Opt. 7, 745 (1968). 